\title{
On the Delta Plus Variant of SARS-CoV-2
}

\author{
Selia Chowdhury and Mehedi Hasan Bappy
}

\section{ABSTRACT}

\begin{abstract}
The unprecedented consequences brought by the COVID pandemic are still going on, the virus hasn't been tamed yet. It is evolving through mutations to consistently being a risk to public health. Recently, the Delta variant has been declared as the variant of concern by the World Health Organization (WHO). In this article, a subvariant of Delta known as Delta Plus has been presented to provide a relevant foundation for future research works. The evolution, pathogenesis, associated symptoms, suggested prevention and treatments, vaccine efficacy, and current trends of transmission of Delta Plus variant of SARS-CoV-2 are reviewed and discussed.
\end{abstract}

Keywords: COVID-19, delta, delta plus, mutation, epidemiology, management, vaccine efficacy.

\section{INTRODUCTION}

The world has seen an unprecedented transmission and mortality due to COVID-19 virus. Millions of people have suffered immeasurable losses, be it physical, mental, financial, or else. Since the beginning of the outbreak, the virus has been going through mutations to change itself and making the task of the health professionals and researchers a lot harder. A total of ten principal variants of SARS-CoV-2 have been reported so far. Among them, Alpha, Beta, Gamma, and Delta are termed as Variants of Concern (VOC) based on their significance to global public health [1]. Currently, Lambda and $\mathrm{Mu}$ are designated as Variants of Interest (VOI) due to their lesser significance compared to VOCs [2]. Delta variant was first reported in India and recently it has emerged as the dominant variant across the world; almost $100 \%$ of the current cases in most of the countries are consequences of this variant [3]. Delta variant is more infective as well as it is causing higher transmissibility compared to all other variants. Delta causes significant number of breakthrough infections in vaccinated individuals [4]. A sublineage of Delta established as "Delta Plus" has been reported to be a health concern by Public Health England [5]. They claimed that this muted variant was detected in six genomes from India till June 7, 2021. It should be noted that the Delta plus variant possesses the K417N mutation in the receptor-binding domain (RBD) of the spike protein. Most of the vaccines against COVID use the spike protein to trigger the body immune system, thus, a mutation in the spike protein can change the way the vaccines work and deteriorate the protection against the coronavirus [6]. More recently, another "plus" variant of Delta (AY.4.2) is
Submitted : November 6, 2021

Published : December 24, 2021

ISSN: $2593-8339$

DOI: $10.24018 /$ ejmed.2021.3.6.1134

\section{S. Chowdhury*}

Dhaka Medical College, Dhaka, Bangladesh.

(e-mail: chowdhury.selia@gmail.com)

M. H. Bappy

University of Iowa, Iowa City, IA, USA.

*Corresponding Author triggering serious concern across UK and USA. UK reported that this variant spiked to $11 \%$ of the current total COVID cases while it spread to at least 8 states in the USA [7], [8]. Based on what is known so far, Delta Plus is considered highly infectious. The total number of Delta Plus variants infections is not very high until now, however, new cases are being reported every day in different countries around the world. The strain has already become a grave concern for additional COVID-19 waves in different countries of the world. It is gradually spreading throughout the world making it a headache to the researchers and health professionals.

\section{Delta Plus Variant: Mutation And Epidemiology}

In India, samples of variant Delta (B.1.617.2) were detected for the first time in October 2020. There are 13 mutations in the B.1.617.2 genome that cause changes in the amino-acid sequences of the encoded proteins. Four specific mutations located in the virus's spike protein code are very concerning, they are T478K, D614G, L452R, and P681R [9]. Delta variant has gone through a number of significant mutations in the spike protein and spread into a few subtypes, which are being classified as Delta Plus. The subtypes AY.1, AY.2, AY.3, and AY.4 are referred to as Delta Plus. They are structurally very analogous to the original Delta variant, but it contains a few changes. Delta Plus has a K417N mutation in the spike protein which is a lysine-to-asparagine substitution at position 417 [9]. The spike protein of this variant, according to Becerra et al. has the same LCRs as the SARS-CoV-2 Delta spike protein [10]. According to [11], The Delta and Delta Plus variants have distinct mutation patterns, and the Delta Plus is not just the Delta variant with 
K417N substitution. They reported that, in comparison to the Delta variant, the Delta Plus contained a higher percentage of high-prevalence mutations (20\%). Three specific mutations (A222V, G142D, and T95I) in spike protein occurred at a higher fraction in the Delta Plus than in the Delta. Another three mutations in the spike protein are unique to the Delta Plus variant (V70F, K417N, and W258L). Additionally, they identified a novel alteration in ORF1a (A1146T); this was distinctively present in the Delta Plus variant with approximately $58 \%$ prevalence. They showed that five mutations (T95I, A222V, G142D, R158G, and K417N) are markedly more frequent in the Delta Plus than in the Delta variant.
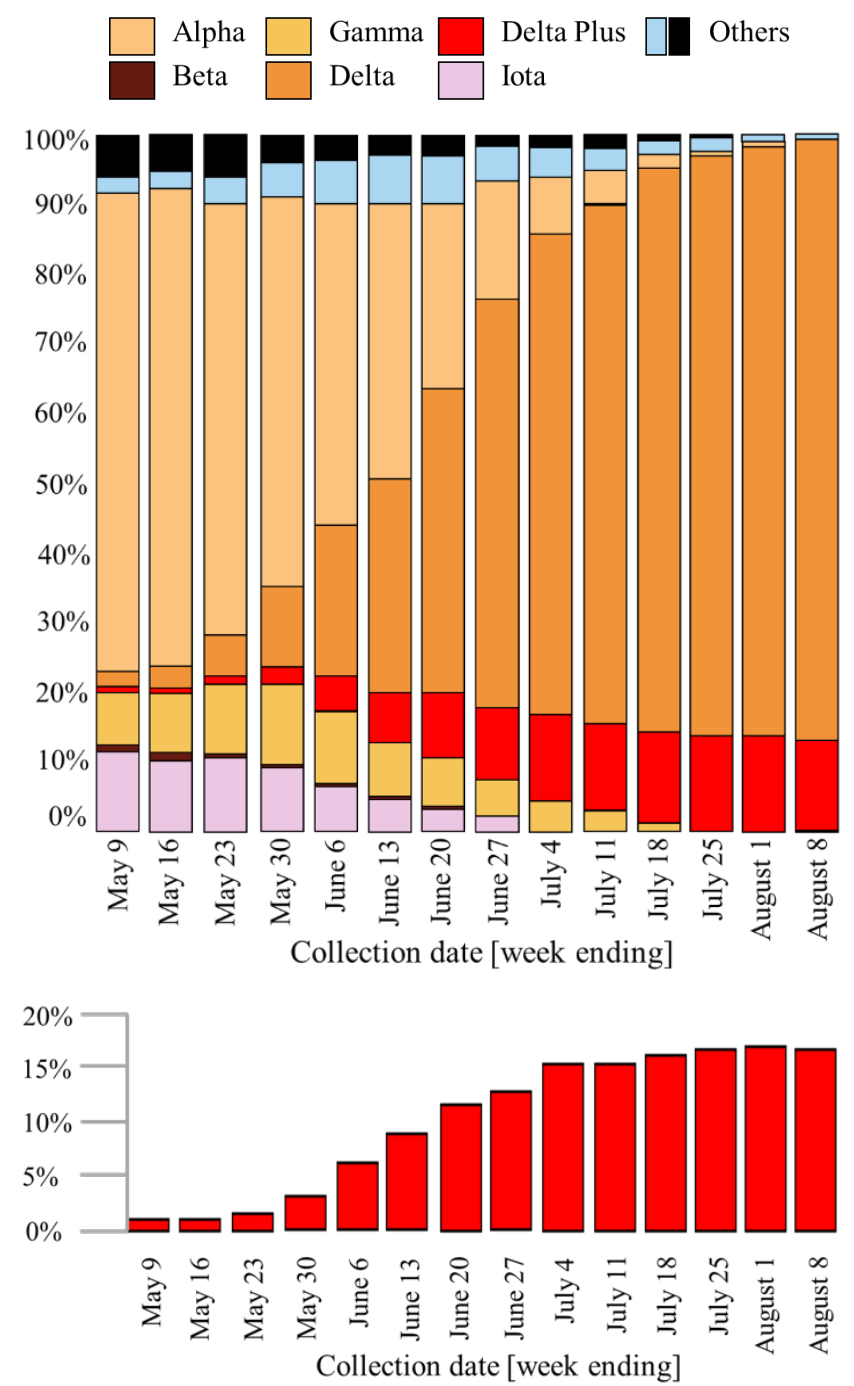

Fig. 1. Distribution of SARS-CoV-2 strains in the United States between the weeks ending on May 9, 2021, and August 8, 2021. The bottom figure shows only the Delta Plus statistics.

The Delta Plus variant had originated in India but it spread through numerous countries including the United States. To learn more about this variant's movement inside the United States, Kannan et al. linked the initial sequences of the Delta Plus variant from various parts of the United States [11]. Using this information up to June 22, 2021, they generated a Sankey diagram by phylogenetic analysis paired with sequence-acquisition which shows how Delta Plus moved through the USA. As of August 17, 2021, the Delta Plus variant has been transmitted to people in more than 40 US states, currently, it has spread to all 50 states. The AY.3 strain has been the most dominant Delta Plus variant accounting for more than $12 \%$ of the total cases while the Delta variant accounts for about $86 \%$ of the cases. The other two variants AY.1 and AY.2 are not very dominant, and they make about 0.1 and $0.3 \%$, respectively, of the total cases [12]. Fig. 1 shows the distribution of variants and their evolution in the United States. The increase of AY.3 is very prominent which calls for taking precautionary measures against the Delta plus sublineages.

According to studies, this variant is quicker and effortless than the original strain of SARS-CoV-2. The transmission rate of Delta and its sublineages are almost twice faster than the original SARS-CoV-2 strain [13]. AY.4 is even more contagious than original Delta, with approximately $15 \%$ more infection rate [14]. According to structural analysis, mutations change the sidechain conformation, weakening antibody interactions [11]. The mutation at K417 is important because it interacts with the ACE2 receptor protein, which enables the virus to infect human cells in organs such as the heart, kidney, lung, heart, and intestines [15]. When the spike protein encounters ACE2, it switches from a "closed" to an "open" state, allowing it to attach and infect the cell more successfully. Other highly transmissible and antibodyresistant mutations follow the same route [5].

According to two different studies, patients who were infected with the Delta variant were more likely to be admitted to the hospital than those who were infected with the Alpha or original viral strains [16], [17]. Breakthrough infections are significantly less common than infections in unvaccinated individuals, but anyone infected with the Delta, including fully vaccinated people who have symptomatic breakthrough infections, can spread it to others. Nonetheless, unvaccinated persons are at the greatest danger of infection since they are much more prone to catch and transmit the virus. In contrast to previous variants, which generally generated less virus in the bodies of fully vaccinated yet infected people than in people who are not vaccinated, the Delta variant generates a substantially high quantity of virus in both fully vaccinated and unvaccinated individuals [13]. However, much like the other variants, the amount of virus released by Delta breakthrough infections decreases faster in fully vaccinated persons than in unvaccinated persons. Therefore, it is evident that people who have been fully immunized are likely to be infectious for a shorter period of time than those who have not been fully immunized.

\section{Clinical Manifestations \& Treatment}

While shortness of breath, cough, and other lung issues are the most common symptoms of COVID-19, the recent variants and subvariants associate other symptoms like headache, sore throat, runny nose, and fever [18]. Other symptoms may include fatigue, muscle or body aches, loss of taste or smell, nausea or vomiting, and diarrhea [18], [19]. Existing treatment for COVID should apply for Delta Plus variant cases, which should be done on case by case basis. Most people who become sick should be able to recover at home by getting proper rest, staying hydrated, and taking medications (acetaminophen) to relieve fever and aches and 
pains. Additionally, the FDA has approved COVID-19 and additional drugs for persons who have been hospitalized to slow the progression of COVID-19 in people who are not hospitalized but are at risk of developing severe disease [20].

The FDA granted emergency use authorization to three monoclonal antibody treatments: a combination of casirivimab and imdevimab, a combination of bamlanivimab and etesevimab, and sotrovimab [20,21]. These treatments are approved for non-hospitalized adults and children over age 12 with mild to moderate COVID-19 symptoms who are at risk for developing severe COVID-19 or being hospitalized for it. These treatments must be administered intravenously as soon as symptoms appear, and they can help to lessen the likelihood of hospitalization and emergency department visits. Monoclonal antibody therapy lowered the risk of death by 20 percent in those who had not developed their own antibodies against the SARS-CoV-2 virus [20]. For people hospitalized with COVID symptoms, doctors may also use Dexamethasone, Tocilizumab, Remdesivir, Baricitinib combined with Remdesivir, and anticoagulation drugs based on the condition of the patients [20].

Because of the specific mutations in the Delta variant, therapeutics such as monoclonal antibody therapies may be less successful. Planas et al. compared this strain to other SARS-CoV-2 strains by testing its sensitivity to monoclonal antibodies and antibodies found in sera from people who had recovered from COVID-19 or who had received a COVID19 vaccination [22]. Some anti-NTD and anti-RBD monoclonal antibodies, including Bamlanivimab, were unable to neutralize the Delta variant, and these antibodies had poor binding to the spike protein. They claimed that the Delta variant's propagation is linked to an avoidance from antibodies that target spike protein's nonRBD and RBD epitopes. Furthermore, antibodies developed by the body in response to a natural infection or a COVID-19 vaccination may not be as effective against the Delta variant as they were against the original strain.

\section{VACCINE EFFICACY}

Existing immunizations are effective against the original Delta form, but they have limited efficiency in elderly age groups, especially in individuals who have not been able to establish an efficient immune response or whose protection may deteriorate more quickly. In the symptomatic Delta variant affected patients, after the first dose of Pfizer vaccine, the efficacy rate is only 36 percent while it is 30 percent after the first dose of AstraZeneca. After the second dose, this rate improved to 88 percent for Pfizer and 67 percent for AstraZeneca [23]. In a report by [24], currently authorized three COVID-19 vaccines for use in the United States showed high effectiveness in preventing laboratory-confirmed COVID-19-related hospitalizations. Among adults aged $\geq 65$ years, the effectiveness of full vaccination with mRNA vaccines (Pfizer and Moderna) was $\geq 91 \%$ and of Janssen was $\geq 84 \%$ [24], [25].

Previous studies on the Beta variant acknowledged that K417 mutations aided to evade antibodies and therefore Delta Plus may bypass vaccines and antibodies [5]. However, there have not been enough research or data on the efficacy of vaccines against the Delta plus variants, a detail evidence based study with large enough population size should be performed to determine the efficacy of the vaccines against each lineage of the Delta variant. According to CDC, vaccines are still quite successful in keeping people out of hospitals and preventing mortality against Delta and its subvariants [13]. They said that fully vaccinated patients with breakthrough infections from these genotypes tend to be infectious for a shorter amount of time and recommend everyone to get vaccinated and wear masks indoors in public spaces to reduce the spread of this variant.

\section{CONCLUSION}

Through mutation, the virus can improve itself infecting people or can help the virus to escape the neutralizing antibodies through mutations in the spike gene. Mutations can either weaken or strengthen the virus enabling it to propagate faster or cause more infections [11]. The Delta variant and its lineages can transmit the disease almost twice as fast as the original strain of SARS-CoV-2, and the severity may also be greater [13]. However, the Delta plus variants have not yet made any overwhelmingly damaging impact compared to the other variants [12], [13]. As more data are available on these variants, more studies are being performed to better understand the variants and their impact on the transmission, hospitalization, efficacy of vaccines, and fatality. We hope that there will be enough research on Delta Plus to successfully avoid any damaging consequence that they and their successive mutants can inflict.

\section{REFERENCES}

[1] Chowdhury S, Bappy MH, Chowdhury S, Chowdhury MS, Chowdhury NS. Current Review of Delta Variant of SARS-CoV-2. European Journal of Medical and Health Sciences. 2021; 3(6): 23-9.

[2] Tracking SARS-CoV-2 variants [Internet]. [cited 2021 Dec 2]. Available from: https://www.who.int/en/activities/tracking-SARSCoV-2-variants/

[3] GISAID - hCov19 Variants [Internet]. [cited 2021 Dec 2]. Available from: https://www.gisaid.org/hcov19-variants/

[4] CDC. What you need to know about variants [Internet]. 2021 [cited 2021 Dec 2]. Available from: https://www.cdc.gov/coronavirus/2019ncov/variants/variant.html

[5] Roy B, Roy H. The Delta Plus variant of COVID-19: Will it be the worst nightmare in the SARS-CoV-2 pandemic? Journal of Biomedical Sciences. 2021; 8(1).

[6] Kyriakidis NC, López-Cortés A, González EV, Grimaldos AB, Prado EO. SARS-CoV-2 vaccines strategies: a comprehensive review of phase 3 candidates. NPJ Vaccines. 2021; 6(1).

[7] Public Health England. SARS-CoV-2 variants of concern and variants under investigation in England. technical briefing 12. 2021.

[8] Alexander A. A potentially faster-spreading Delta variant, AY.4.2, has been spotted in 8 states [Internet]. CBS News. 2021 [cited 2021 Dec 2]. Available from: https://www.cbsnews.com/news/covid-delta-plusvariant-ay-4-2-states/

[9] Wikipedia contributors. SARS-CoV-2 Delta variant [Internet]. Wikipedia, The Free Encyclopedia. 2021 [cited 2021 Dec 2]. Available from: https://en.wikipedia.org/w/index.php?title=SARS-CoV2 Delta variant\&oldid $=1058079794$

[10] Becerra A, Muñoz-Velasco I, Aguilar-Cámara A, Cottom-Salas W, Cruz-González A, Vázquez-Salazar A, et al. Two Short Low Complexity Regions (LCRs) are Hallmark Sequences of the Delta SARS-CoV-2 Variant Spike Protein. 2021.

[11] Kannan SR, Spratt AN, Cohen AR, Naqvi SH, Chand HS, Quinn TP, et al. Evolutionary analysis of the delta and Delta Plus variants of the SARS-CoV-2 viruses. Journal of Autoimmunity. 2021; 102715.

[12] CDC. COVID Data Tracker. [Internet]. 2020 [cited 2021 Dec 2]. Available from: https://covid.cdc.gov/covid-data-tracker/ 
[13] CDC. Delta variant: What we know about the science. [Internet]. 2021 [cited 2021 Dec 2]. Available from: https://www.cdc.gov/coronavirus/2019-ncov/variants/deltavariant.html

[14] Burn-Murdoch J. New Delta descendant may be more infectious than its ancestor. Financial Times [Internet]. 2021 [cited 2021 Dec 2]; Available from: https://www.ft.com/content/flec9d5d-9e02-4cc495e7-1dcbb1844d43

[15] Chowdhury S, Bappy MH, Chowdhury S, Chowdhury MS, Chowdhury NS. COVID-19 induced cardiovascular complications and recent therapeutic advances. European Journal of Medical and Health Sciences. 2021; 3(6): 17-22.

[16] Ong SWX, Chiew CJ, Ang LW, Mak TM, Cui L, Toh MPH, et al. Clinical and Virological Features of SARS-CoV-2 Variants of Concern: A Retrospective Cohort Study Comparing B. Clinical Infectious Disease. 2021; 1: 1.

[17] Fisman D, Tuite A. Progressive Increase in Virulence of Novel SARSCoV-2 Variants in Ontario. Canada; 2021.

[18] CDC. Symptoms of COVID-19. [Internet]. 2021 [cited 2021 Dec 2]. Available from: https://www.cdc.gov/coronavirus/2019ncov/symptoms-testing/symptoms.html

[19] Miller K. What is the Delta Plus variant? Here's what experts know about it so far. [Internet]. 2021 [cited 2021 Dec 2]. Available from: https://www.prevention.com/health/a37221697/what-is-delta-plusvariant-covid-19/

[20] Treatments for COVID-19. [Internet]. 2021 [cited 2021 Dec 2]. Available from: https://www.health.harvard.edu/diseases-andconditions/treatments-for-covid-19

[21] Information on COVID-19 treatment, prevention and research. [Internet]. [cited 2021 Dec 2]. Available from: https://www.covid19treatmentguidelines.nih.gov/

[22] Planas D, Veyer D, Baidaliuk A, Staropoli I, Guivel-Benhassine F, Rajah MM, et al. Reduced sensitivity of SARS-CoV-2 variant Delta to antibody neutralization. Nature. 2021.

[23] Lopez Bernal J, Andrews N, Gower C, Gallagher E, Simmons R, Thelwall S, et al. Effectiveness of Covid-19 vaccines against the B. New England Journal of Medicine. 2021; 1: 617.

[24] Moline HL, Whitaker M, Deng L, Rhodes JC, Milucky J, Pham H, et al. Effectiveness of COVID-19 vaccines in preventing hospitalization among adults aged $\geq 65$ years - COVID-NET, 13 states, FebruaryApril 2021. MMWR Morb Mortal Wkly Rep. 2021;70(32):1088-93.

[25] Tenforde MW, Self WH, Naioti EA, Ginde AA, Douin DJ, Olson SM, et al. Sustained effectiveness of Pfizer-BioNTech and Moderna vaccines against COVID-19 associated hospitalizations among adults-United States, March-July 2021. Morbidity and Mortality Weekly Report. 2021; 70(34): 1156. 